Revista de Dialectología y Tradiciones Populares, vol. LXXI, n. ${ }^{\circ}$ 1, pp. 39-48, enero-junio 2016, ISSN: 0034-7981, eISSN: 1988-8457, doi: $10.3989 /$ rdtp.2016.01.001.03

\title{
Tiempos de colaboración: performances del conocimiento urbano
}

\author{
Times of Collaboration: \\ Performances of Urban Knowledge
}

\author{
Montserrat Cañedo Rodríguez \\ Universidad Nacional de Educación a Distancia \\ mcanedo@fsof.uned.es
}

\section{RESUMEN}

En esta contribución me propongo partir de una experiencia concreta de lo que designaré como ejemplo de "colaboración experimental", para discutir algunos aspectos relacionados con la producción del conocimiento antropológico en la ciudad de hoy día. La descripción etnográfica de un proyecto sobre las infraestructuras de Madrid desarrollado en el espacio de un centro cultural de la ciudad me permitirá, no tanto delimitar la figura y el valor en abstracto del experimento en antropología —un debate que desbordaría el limitado espacio de este texto-, cuanto utilizar un proyecto de investigación antropológica algo diferente al habitual para dar otra vuelta de tuerca a la reflexión que acompaña desde siempre a la disciplina, manifestando no obstante en cada momento y lugar un carácter tópico: la reflexión sobre la legitimidad del conocimiento antropológico y sobre las condiciones prácticas de su producción. El topos de este texto es la ciudad; o quizás, siendo más precisos, la metrópoli occidental contemporánea, donde en este caso la antropóloga viene a ser lo que con un sentido cada vez más difuso se llama todavía antropóloga "en casa".

Palabras clave: Co-producción de conocimiento; Experimentación; Medialab; Mercamadrid.

\section{SUMMARY}

The article takes a specific experience of what I shall suggest is an example of "experimental collaboration" to discuss aspects of the production of anthropological knowledge in today's cities. The ethnographic description of a project on Madrid's infrastructures developed in one of the city's cultural centers will allow me, not so much to define the abstract value and nature of the experiment in anthropology — a debate that would exceed the limited space of this text—as to use a somewhat different kind of anthropological project to give a new twist on a reflection that has always accompanied the discipline, despite its topical character in a given time and place, namely reflection on the legitimacy 
of anthropological knowledge and on the practical conditions of its production. The topos of this text is the city or, perhaps, more precisely, the contemporary Western metropolis, where, in this case, anthropology emerges as what is still called, though increasingly diffusely, anthropology "at home."

Key words: Co-Production of Knowledge; Experimentation; Medialab; Mercamadrid.

A comienzos del 2011, varios antropólogos y antropólogas entre los que me incluía nos encontrábamos a mitad del desarrollo de un proyecto de investigación del Departamento de Antropología de la UNED, financiado por el Plan Nacional de I+D+i, en el marco del cual realizábamos entonces nuestras etnografías sobre la ciudad de Madrid. ${ }^{1}$ Con Practicemad —el nombre del proyecto, que abreviábamos por su acrónimo- pretendíamos aproximarnos al fenómeno de la emergencia cultural urbana, a través de localizaciones empíricas variadas y heterogéneas transversalmente a las cuales intentábamos, no obstante, tender hilos comunes: la centralidad cultural de la novedad y las transformaciones en las formas de la subjetivación asociadas a esta o el rol de las mediaciones tecnológicas en ambos procesos, entre otras cosas. ${ }^{2}$ Un elemento común con el que nos topábamos en nuestros campos era la proliferación de encuentros con personas y colectivos - activistas, asociaciones de vecinos, centros culturales o consultoras- que compartían con nosotros un mismo objetivo: producir conocimiento sobre el Madrid contemporáneo, valga decir en su dimensión cultural. Partiendo de una inquietud disciplinar, diría que de época — de la que no podíamos menos que hacernos eco, máxime cuando trabajábamos sobre una ciudad que era la nuestra-, aquellos sujetos que poblaban nuestros campos empíricos encajaban difícilmente en el clásico rol de "informantes". La dimensión política de nuestra tarea como investigadores, entendida en sentido amplio como política del conocimiento, se traducía así en una inquietud compartida acerca de cuáles debían ser los métodos y los productos de nuestro trabajo con ellos.

El formato académico configuró en cierto modo algunos de los primeros encuentros: nuestro proyecto incluía en su diseño a un puñado de interlocutores en determinados espacios de la urbe, ${ }^{3}$ algunos de los cuales fueron espontáneamente revelándose como un foco de atracción, quizás porque quintaesenciaban esos aires de familia que encontrábamos entre sus reflexiones y las propias. ${ }^{4}$ Uno de esos lugares era el MedialabPrado, un tipo novedoso de institución cultural extendido por muchas ciudades des-

\footnotetext{
${ }^{1}$ Prácticas culturales emergentes en el Nuevo Madrid (MEC CSO2009-10780). I.P. Francisco Cruces.

${ }^{2}$ Las más recientes publicaciones colectivas derivadas de ese proyecto y de su continuación en el titulado Madrid Cosmópolis. Prácticas emergentes y procesos metropolitanos (MEC CSO201233949) son: Grupo Cultura Urbana UNED 2016 (a) y (b).

${ }^{3}$ Las llamadas EPOS (entidades promotoras observadoras) en la jerga de los proyectos I+D+i.

${ }^{4}$ Algunos colegas nos avisaban de un probable sesgo etnocéntrico en nuestro interés por esos entornos, como si éste se derivase de que sus miembros replicaban nuestras propias inquietudes y pre-juicios (de clase, de edad, de formación escolar, de hábitat residencial, etc.) Sin embargo, en una disciplina ya bien sacudida por los sucesivos "giros" textuales, ontológicos, etc., ya nadie creía en aquello de la "objetividad" entendida stricto sensu como una distancia. Más bien, si acaso, estaba claro que había que inventarse otros lenguajes prácticos para construirla, que pensamos nos obligaban a asumir los retos que la proximidad a esas personas, en cierto sentido pares epistémicos, nos planteaba.
} 
de comienzos del 2000, como híbrido singular entre — jal menos! — las prácticas del curador, de la activista, del profesor-estudiante, de la científica profesional o amateur, del fan del DIY, etc., alrededor de una metodología centrada en la colaboración y de ciertos tópicos temáticos, amplios pero característicos (el urbanismo, la ciencia ciudadana, el código abierto, etc.). ${ }^{5}$ Desde el Medialab nos hicieron llegar una invitación a presentarnos a la convocatoria de aquel año de su programa Visualizar. Un programa centrado en la llamada visualización de datos, esto es, en la idea de generar —en un formato de taller con colaboradores interesados en los proyectos aceptadosformatos visuales y narrativos a partir de la enorme cantidad de información, cuantitativa fundamentalmente, que producen las instituciones urbanas. El tema de 2011 eran las infraestructuras. ${ }^{6} \mathrm{Y}$, precisamente, mi ventana etnográfica a la cosmópolis madrileña estaba en el Mercamadrid, el mercado mayorista de distribución alimentaria de la capital. Una institución que venía produciendo con sistemática periodicidad datos muy interesantes - y muy desconocidos - sobre los orígenes geográficos de los alimentos que consumen los madrileños. Ocupada como estaba en ensayar si la artesanía de la etnografía podía constituir algún retrato de la globalización alimentaria de Madrid y consciente de la enorme opacidad del sistema experto que encapsulaba ese nodo central —el mercado mayorista - del abastecimiento urbano, pensé que podía ser una buena idea presentar algo.

Mis motivos eran un tanto indefinidos y de orden distinto, aunque todos me parecían importantes: hacer etnografía en un entorno que podía definirse como "emergente»; tomarme en serio el trabajo con una EPO más allá de declaraciones programáticas; dar forma, junto a un todavía desconocido grupo de no antropólogos, a un objeto, en un interesante doble sentido: un objeto de estudio, pero también un objeto propiamente material como el producto mismo de la colaboración, que fuera más allá de la habitual forma textual; experimentar con un activismo vagamente definido desde los presupuestos de una ciencia ciudadana. El catalizador de la decisión fue, no obstante, un encuentro con César, muy familiarizado con las convocatorias del Medialab y ejemplo perfecto de uno de los perfiles de sus habituales: tecnólogo de formación y profesión, pero también humanista en sus inquietudes, habituado al método de los proyectos en colaboración y con un entusiasmo desbordante. Juntos preparamos un proyecto, en el rol de "promotores": una suerte de cartografía crítica que dibujaba insólitos espacio-tiempos del abastecimiento urbano. ${ }^{7}$

El proyecto fue uno de los diez seleccionados de entre los más de cincuenta presentados, en parte —supongo- por el interés que habría despertado entre los evaluadores, pero sin duda también por un asunto de oportunidad política: nuestro proyecto, a diferencia de otros igualmente ajustados a la convocatoria, se centraba en una institución local infraestructuralmente central en la urbe. El vínculo de las actividades desarrolladas en el Medialab con Madrid —y con su Ayuntamiento- era fun-

\footnotetext{
${ }^{5}$ Una etnografía de alguno de estos entornos, incluido el Medialab-Prado, en Fernández 2015. Véase también Estalella 2015.

${ }^{6}$ http://medialab-prado.es/article/visualizar10_comprendiendo_las_infraestructuras [Acceso 07/ 03/16]

${ }^{7}$ http://medialab-prado.es/article/visualizar11_proyectos_y_comunicaciones_seleccionadas [Acceso $07 / 03 / 16]$
} 
damental para la justificación, siempre un tanto problemática, de una institución cultural hasta esa fecha tan atípica. Nuestro proyecto, simplemente, encajaba con ese otro interés. Esto, que no tiene nada de particular, pero que sin embargo se le reveló a la antropóloga como una novedad con respecto a otros condicionamientos institucionales más invisibles (pongamos en el propio marco de su proyecto académico) a los que simplemente estaba más habituada, me permite subrayar una obviedad: cómo las condiciones materiales de la producción de conocimiento y lo que legítimamente cuenta en cada caso como una agenda de problemas interesantes toman parte en la forma de cualquier objeto de estudio.

Una vez aceptado, el proyecto requería el reclutamiento de colaboradores, en un proceso ya formalizado en tiempo y forma y bien ensayado en el Medialab, que se extendió a lo largo de dos meses durante la primavera de 2011. Creo, sin embargo, que no fue sino en el seminario inicial al taller, celebrado el primer día del mismo y donde cada uno de los promotores tenía que presentar al auditorio su proyecto, ${ }^{8}$ cuando la mayor parte de un público difusamente interesado en participar como colaborador fue centrando su interés en uno u otro de los presentados, entre ellos el nuestro. ${ }^{9}$ En esta presentación pública tuve una sensación que me acompañó durante todo el experimento como promotora: el proyecto necesitaba frecuentemente de una narrativa que convenciera a un auditorio — de una escala diferente en cada momento- de su interés, siempre de algún modo necesitado de justificación y en competencia con otros. Esto, que por un lado despertó muchas veces el recelo de tener que dedicarnos en demasiadas ocasiones a "vender" nuestra idea, planteó también el reto de mantener abierta la pregunta por la relevancia del proyecto y su para quién. La "colaboración" no estaba presupuesta. La antropóloga tendría que negociar su objeto en un proceso donde iba forzosamente a aprender a ceder el control del mismo proceso de su construcción. Y no se trata obviamente de que en el contexto académico los proyectos no necesiten ser justificados, pero los tiempos son otros y la aprobación inicial abre un periodo de gracia en el que la investigadora puede confiar en la legitimidad de su dispositivo. Por otra parte, en el "campo" de la etnógrafa — por citar otro locus canónico- el proyecto como tal no necesita exactamente ser compartido (y justificado), al menos en términos generales. Pero en este experimento, la reiteración acelerada de las "exposiciones" y las demandas de justificación eran tales que convertían el asunto en algo acuciante y en una fuente de estrés que, sin embargo, diría que funcionaba, en esa infraestructura epistémica que fue en sí misma el taller, como una condición de posibilidad de un cierto ritmo y un estilo en la producción de conocimiento. ${ }^{10}$ Lo veremos más adelante.

${ }^{8} \mathrm{http}: / /$ medialabprado.es/article/visualizar11_proyectos_y_comunicaciones_seleccionadas [Acceso $07 / 03 / 16]$

9 Agradezco a Ester Gisbert, Beatriz García, Fabio Silli, David Rodríguez, Sara Alvarellos, Javi Esquillor, Martina Minnucci, María Olmeda, Víctor Díaz, Carlos Panero y Gorka Cortázar su disposición a colaborar con César García y conmigo en este proyecto. Agradezco también las discusiones que mantuvimos en el Medialab, que me inspiraron parte de las reflexiones vertidas en este texto.

${ }^{10}$ Sería interesante el estudio de la relación entre algunos condicionamientos omnipresentes en estos nuevos entornos de producción de conocimiento y otros que han ido produciendo en la academia los nuevos dispositivos de accountability (Strathern 2000, Gill 2010). 


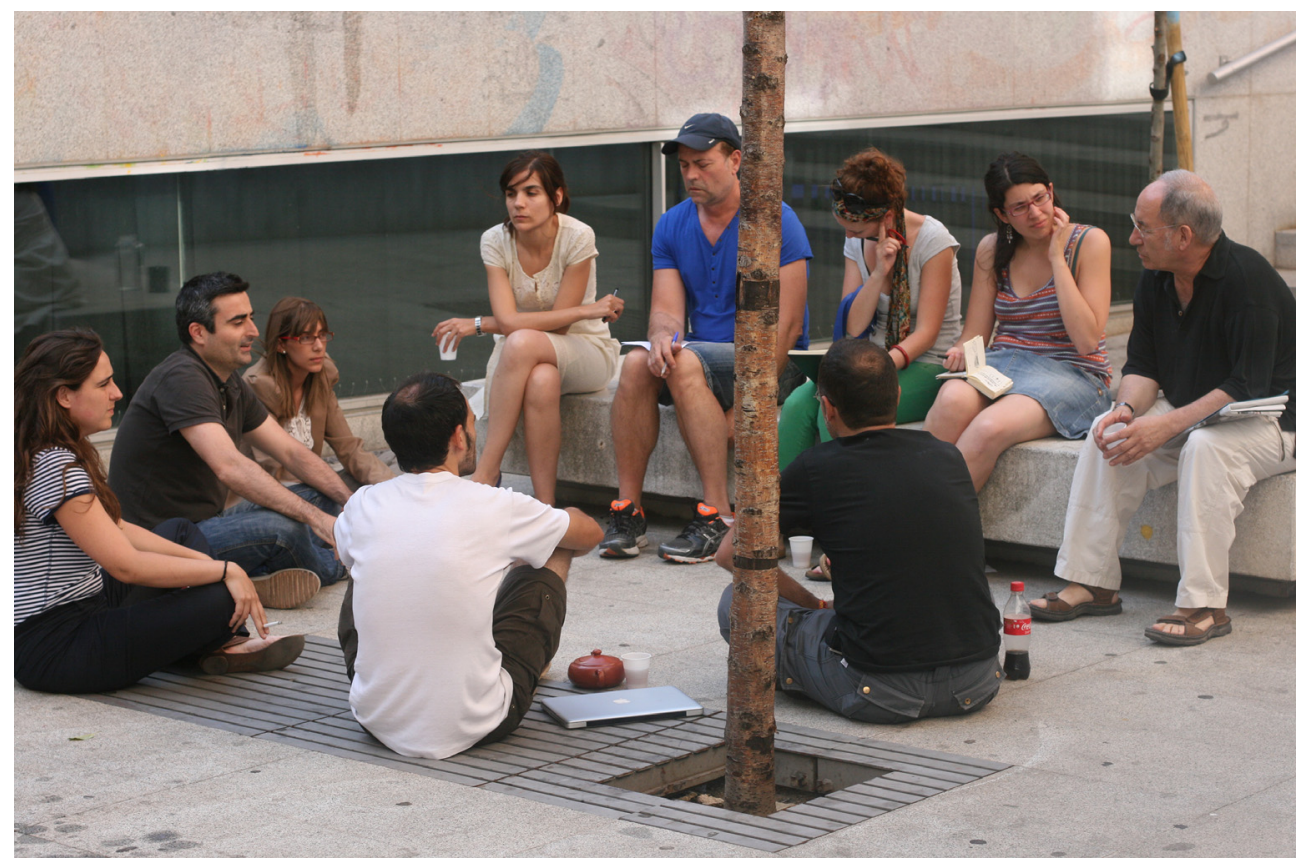

IMAGEN 1.-Parte del grupo en una charla con integrantes de SlowFood España y mercadodeabastos.org. Autor: Medialab-Prado. Licencia de la imagen: https://creativecommons.org/licenses/by-nc-sa/2.0/

El proyecto se llevó a cabo con el concurso de once colaboradores, además de los dos promotores: varios arquitectos, algún artista, un par de programadores, todos ellos con trayectorias, experiencias y saberes diversos. Tras el mencionado seminario inicial —donde varios ponentes, la mayor parte llegados desde el extranjero, dieron charlas y presentaron proyectos afines a los que se iban a desarrollar allí; y donde también se presentaron los "profesores" que servirían de guías y el calendario que marcaría el desarrollo del taller, puntuado de sucesivas puestas en común ante todo el auditorio- nuestro grupo empezó a trabajar en el espacio asignado: una gran mesa provista de rotuladores y un rollo de papel de gran formato para anotaciones (huelga decir que cada uno se traía de casa la herramienta principal: su portátil). El diseño de la interacción de ese dispositivo epistémico ${ }^{11}$ que en sí mismo era el taller, resultaba muy eficaz y estaba muy ensayado en el Medialab, siendo familiar también al menos a gran parte de los participantes, que ya conocían esas dinámicas de trabajo. A mí, sin embargo, se me hizo exótico y, a mi pesar, a ratos fastidioso.

Los distintos grupos trabajábamos en una gran sala común, cada uno en su mesa. Las "interferencias" eran constantes: a lo largo de las intensas sesiones diarias (de mañana y tarde), diversas actividades más o menos relacionadas con los tópicos de la

\footnotetext{
${ }^{11}$ Utilizo este concepto de inspiración foucaultiana para incidir en el hecho de que el conocimiento depende siempre de una infraestructura material que ensambla elementos heterogéneos, que por supuesto incluye las ideas y las formulaciones de los objetos de estudio (Agamben 2015).
} 
convocatoria puntuaban el ritmo de trabajo y concitaban a parte del auditorio, que cada cierto tiempo dejaba de ocuparse de su proyecto por un rato. Estas salidas y entradas del trabajo en pequeños grupos hacia otras actividades con otros en una atmósfera general de actividad multitarea eran constantes y, así, no era raro ver cómo el grupo se subdividía en parejas, o tríos o individuos solos, ora trabajando, ora prestando atención a algún ponente o haciendo alguna otra cosa con el ordenador. En ese fluido de actividad incesante y un tanto difusa, puntuada de atractores de la atención y la acción, un vector que marcaba el ritmo colectivo y volvía a recordarnos el objetivo de llevar a cabo los proyectos era el calendario de "tutorías" con los profesores, así como las presentaciones intermedias del estado de los trabajos, que generaban un enorme estrés que culminaba en la apoteosis del día de la presentación final (muy parecido al día del estreno de alguna obra o exposición artística). En el marco de esa actividad incesante y un tanto frenética, donde la perfomance (de la propia posición en el y los grupo(s), del objeto de la colaboración, etc.) era una tarea casi continua porque sujetos y objetos parecían cambiar de forma a cada rato - hasta me cupo alguna vez la posibilidad de que nos olvidáramos del asunto del Mercamadrid-, no resulta extraño que los dispositivos de memoria adquiriesen una presencia central. Así, desde la organización del taller nos animaban a "documentarlo" todo: a dejar constancia visual y escrita de qué se hacía, cómo y por qué; en videos, fotografías, hojas de sala y sofisticados inscriptores de todo tipo, cuya utilidad en el asunto — ¿central? - del desarrollo de los proyectos parecía sin embargo mucho más irrele-

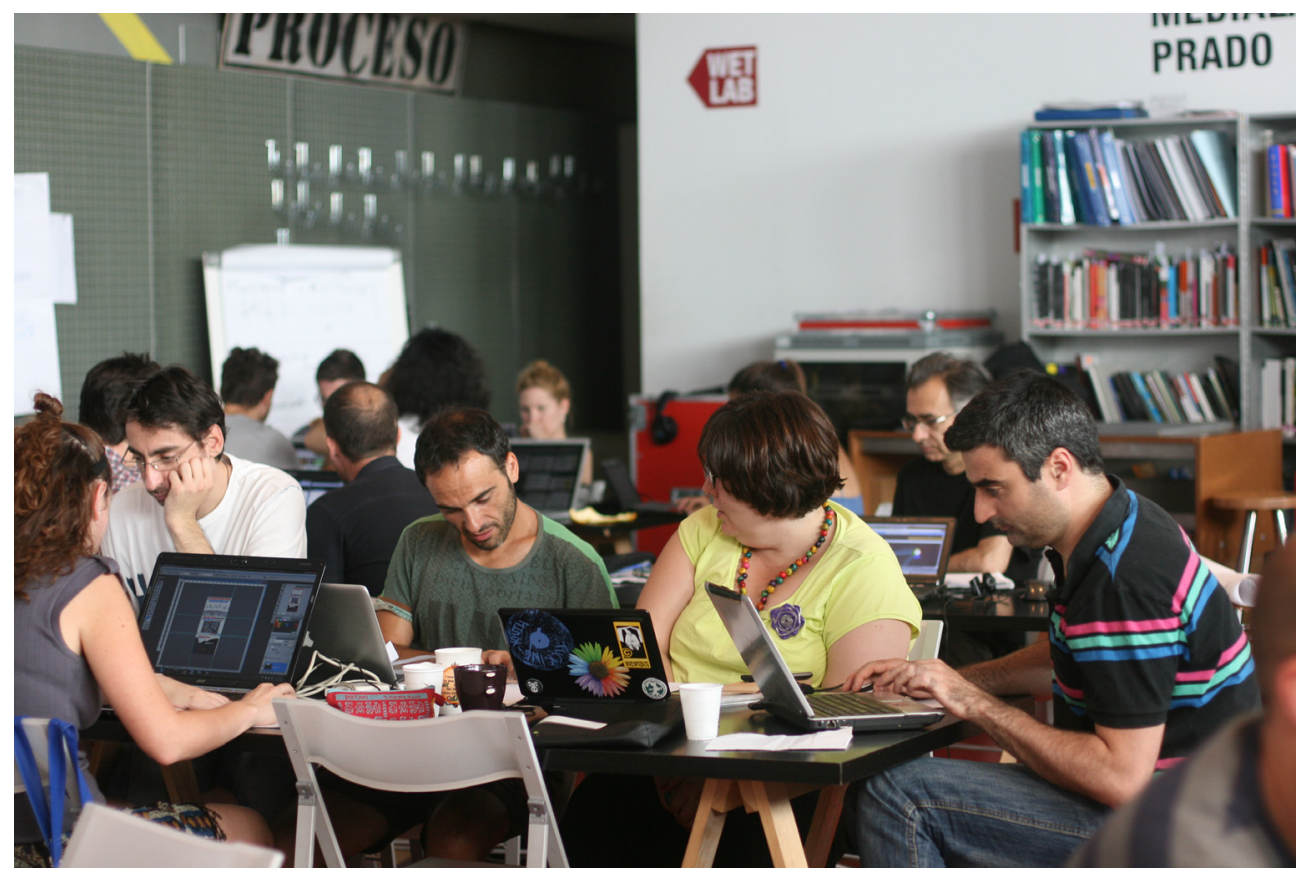

IMAGEN 2.- - Parte del grupo trabajando en el proyecto. Autor: Medialab-Prado. Licencia de la imagen: https://creativecommons.org/licenses/by-nc-sa/2.0/ 
vante, a juzgar por las veces (ninguna) que utilizamos los materiales de otras ediciones, por ejemplo. Como si hubiese que llenar una biblioteca virtual con todos los documentos que en el espacio de la frenética y pura performance nunca habría tiempo de consultar, pero que otorgaban una cierta solidez o permanencia a los objetos... ¿A qué objetos? En realidad esto ya viene a ser una metáfora, o mejor dicho una encarnación, de lo que Latour llama "referencias circulantes" (Latour 2001) ${ }^{12}$.

En ese contexto, nuestro grupo, como los demás, se afanaba por darle forma al objeto material en el que iba a consistir nuestra colaboración. A pesar del entusiasmo colectivo por el proyecto y la intensidad del trabajo derivada también del propio diseño del taller —-quince días de trabajo intensivo—, la colaboración nunca significó que el sentido que cada uno daba a lo que se estaba haciendo fuera similar. Se trató siempre de una colaboración basada en la fricción (Tsing 2013). El proceso de ir decidiendo qué se hacía duró prácticamente el mismo tiempo que el hacerlo. Las discusiones sobre qué hacer y los intentos por llegar a propuestas consensuadas que marcaran la construcción del objeto se revelaron infructuosos. La gente quería dejar de hablar y "empezar a hacer algo" cuanto antes. Así que el modelo clásico, incorporado en el dispositivo epistémico de los proyectos más académicos y que funcionaba para mí al menos como una modelización de lo que debía ser la práctica de la construcción del objeto, aquí se colapsaba. La en algún sentido falta de coherencia no iba a significar, sin embargo, incoherencia, sino algo así como el (re)ensamblaje permanente de la no coherencia (Law 2004).

La concreción principal del proyecto sería una aplicación que proyectara sobre un mapamundi unos puntos de luz de mayor o menor tamaño en función de los datos que la alimentarían (los datos de volumen y procedencia de un grupo seleccionado de alimentos, que tomábamos de Mercamadrid). Pero desde un punto de vista antropológico, se podían hacer más cosas. Por ejemplo, producir algún conocimiento sobre los sentidos locales de esos flujos que la aplicación transformaba en una experiencia visual tan estética como, en cierto modo, fetichista. Así, por ejemplo, todo el equipo se fue a hacer entrevistas al Mercamadrid, cuyos guiones más o menos diseñamos previamente, y algunos de cuyos fragmentos grabados en audio insertamos en el mapa de tal modo que podían escucharse en una navegación libre por parte del potencial usuario (de la aplicación). Todo esto $-\mathrm{y}$ alguna otra cosa que no tengo espacio para contar aquí-, en teoría; en la práctica no diremos que no resultó, pero sí que funcionó más o menos. El problema no fue tanto que nuestro objeto fuese un prototipo aún lejos de su versión terminada (algo que ya estaba previsto), sino que era un prototipo en cierto modo monstruoso, hecho de piezas difícilmente compatibles. El ritmo más lento que exigía construir un relato antropológicamente coherente con retazos de entrevistas y datos de orden cuantitativo chocaba con exigencias de orden distinto, que apelaban también a la coherencia, esta vez de tipo visual y estético, que nuestro objeto tampoco parecía terminar de lograr. Las competencias/incompetencias de unos y otros en las distintas habilidades y saberes implicados en la construcción de nuestro objeto tampoco eran asunto baladí. Curiosamente, el control que

\footnotetext{
${ }^{12}$ De acuerdo a este autor, el objeto "real" (la referencia) no existe como una entidad unificada y exterior a las cadenas de traducciones articuladas que lo enactan (i.e., al propio dispositivo epistémico en cuyo "interior" la referencia circula).
} 
definitivamente la antropóloga perdía toda vez que en el centro del asunto se colocaba cierto know how tecnológico (los programadores fueron en varios momentos los directores del proceso, sobre todo cuando hacia el final nos temimos que "de aquello no fuera a salir nada"), lo volvía a recuperar cada vez que, en pequeño, mediano o gran grupo, era necesaria una narrativa que contase en palabras el proyecto. La distribución disciplinar de las tareas en la colaboración parecía que dejaba un hueco a la antropología, pero pudiera ser que sobre todo como disciplina retórica. Una habilidad con el discurso un tanto incómoda — la de la antropóloga- porque una podía pensar que ese discurso que en cada oportunidad improvisaba no respondía a la coherencia del objeto, sino que se desplegaba como una justificación extrínseca dentro de un particular dispositivo epistémico. Sin embargo, de nuevo, al borde del sinsentido, asomaba otra vez alguna esperanza para la descolocada investigadora: su narrativa conseguía a veces relanzar el proyecto — lo que llevaba a mejorar el prototipo- porque interpelaba una y otra vez al colectivo, proyectando un sentido temporal a futuro que volvía a reunir todas aquellas fuerzas, tendentes por lo demás a la entropía en un entorno híper-estimulado donde los anclajes de la memoria — ¿qué dijimos que estábamos haciendo? - eran débiles. La narrativa ayudaba a que el objeto se mantuviese, más que en su incoherencia en su no-coherencia, y a que existiese como tal objeto en tanto persistiera (re)uniendo a las partes en una colaboración que, en ese sentido - me decía en mis momentos más optimistas —- puede ser exitosa a pesar de que, ciertamente, no siga en absoluto las reglas de lo que sería un objeto bien construido de acuerdo al dispositivo epistémico académico. Este optimismo un poco perplejo se extendió más allá incluso del fin del taller, en lo que luego alguna vez llamamos larga vida del cadáver.

Porque el prototipo resultó, al menos en parte, fallido como objeto material: la visualización de datos era confusa y con dificultad inteligible. Un blog que recogía otros resultados de la colaboración fue, eso sí, un espacio más amigable para la presentación de una no-coherencia. ${ }^{13}$ Pero no por ello el proyecto dejó de ser presentado en sociedad como correspondía, y el imperativo de la consistencia del objeto resultó a medias solventado por el efecto estético y el retórico que proyectaron el objeto y sus autores, con el necesario concurso de la caridad del público. En todo caso, la presentación final de los proyectos no fue sino un momento de celebración de la convocatoria - los saberes intercambiados, las preguntas (auto)formuladas, las relaciones trazadas, los futuros proyectos y el simple disfrute del jintenso! recorrido compartido-, con independencia de las diferentes valoraciones sobre los objetos producidos. Y además, la cosa no acabó ahí. En nuestro caso, las propuestas de continuar trabajando en el prototipo ya al margen de la convocatoria del Medialab se quedaron - hasta la fecha- en meras ideas. El grupo se disgregó, como era de prever, por otros proyectos profesionales y vitales. Pero nuestro prototipo tuvo una vida posterior a su finalización: en dos exhibiciones del Medialab del 2013 y 2015 fue recuperado para volver a ser expuesto, lo cual necesitó y promovió la reunión de parte del equipo. Varios medios de comunicación —el diario Cinco Días y hasta la BBC- contactaron con el centro, y después con los autores, para pedir y difundir más información so-

\footnotetext{
13 https://mercamadridvisualizar.wordpress.com/ [Acceso 07/03/16]
} 


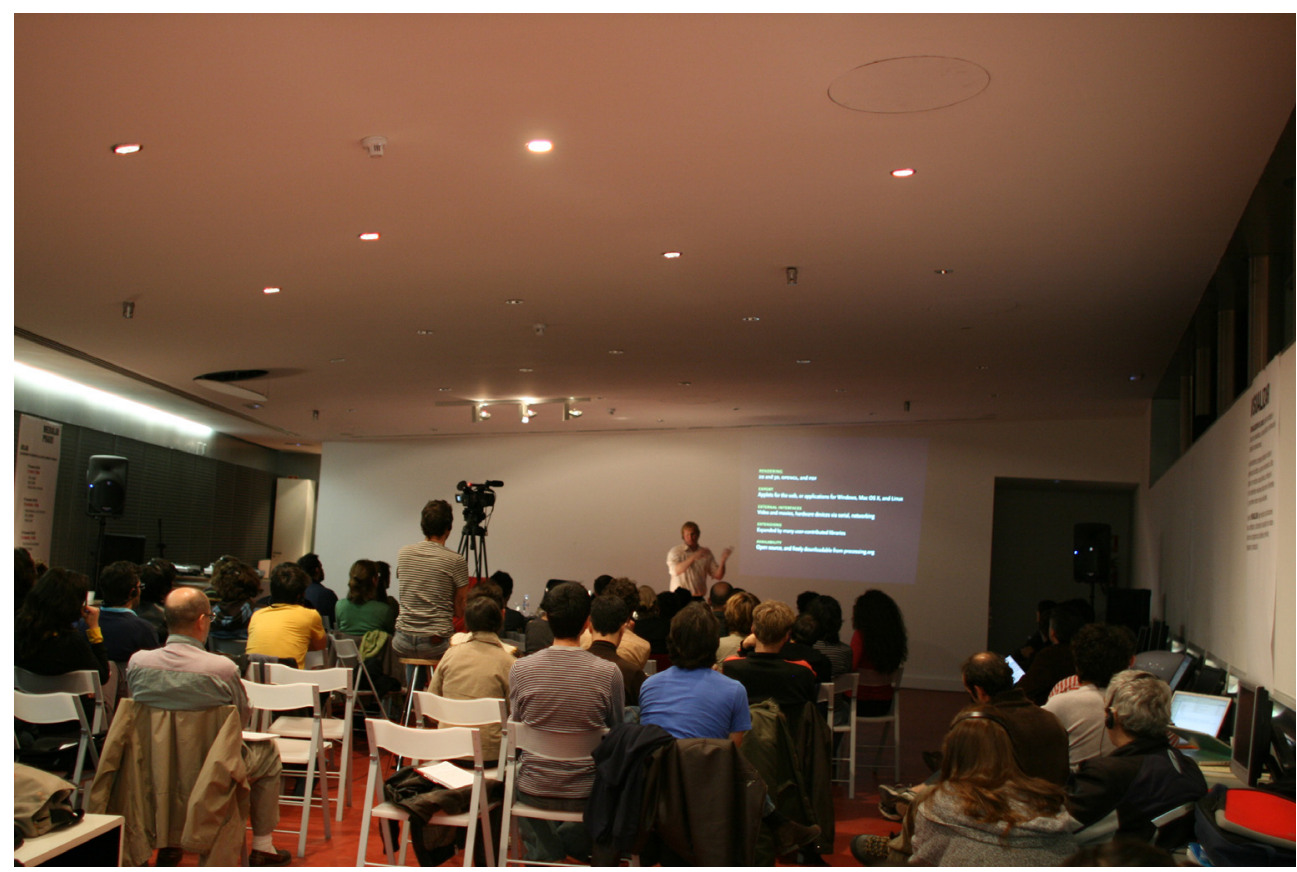

IMAGEN 3.-Exposición de proyectos en el Medialab-Prado. Autor: Medialab-Prado. Licencia de la imagen: https://creativecommons.org/licenses/by-nc-sa/2.0/

bre el proyecto. Diferentes personas y grupos (de investigación académica, activismo ciudadano, e incluso un técnico del departamento de estadística del Mercamadrid) se interesaron por cómo podrían colaborar en el asunto.

Mirando desde cierto lugar —el dispositivo académico de la antropología-, puede decirse que se dibuja hoy día una ecología de la producción y difusión de conocimiento sobre la ciudad que tiende a disolver la artesanía del slow pace etnográfico y el modo en el que se sostienen sobre él la consistencia o la legitimidad epistemológicas, en un retablo de las apariencias sujeto a la "tiranía del momento" (Eriksen 2001) ${ }^{14}$. Paradójicamente, la vida de estos novedosos objetos, que en el marco de aquel dispositivo resultan en todo o en parte fallidos, prueba sin embargo una gran capacidad de abrir otras posibilidades de colaboración en penoso contraste con el corto recorrido de parte de la producción académica — me refiero a esos formatos textuales que no necesariamente alguien lee-. Que abrir posibilidades de colaboración no necesariamente significa — como ilustra este caso- producir conocimiento relevante, o con-

${ }^{14}$ La tesis de Eriksen - sugerente, aunque un tanto unidimensional cuando se proyecta in toto sobre el problema de la temporalidad en la contemporaneidad-sostiene que las tecnologías de la información han contribuido decisivamente a informar una lógica de la aceleración y fragmentación del tiempo que tiene en la "saturación (de actividad) del momento" una de sus notas características. 
sistente, o legítimo, o incluso antropológico, es obvio. Pero lo que este experimento ilumina es precisamente una cara de esa nueva ecología de producción del conocimiento sobre la ciudad que reta a los dispositivos epistémicos más habituales, permitiendo al menos imaginar reformulaciones diferentes del canon. El experimento, así, como un ejercicio de difracción (Haraway 2004) de nuestra infraestructura epistémica. Cómo haya de (re)construirse la relevancia, la legitimidad y todo lo demás en ese humus urbano, es una pregunta de orden epistemológico y político que los antropólogos actuales y sus potenciales colaboradores no pueden soslayar.

\section{BIBLIOGRAFÍA CITADA}

Agamben, Giorgio. 2015. ¿Qué es un dispositivo?. Barcelona: Anagrama.

Eriksen, Thomas Hylland. 2001. Tyranny of the Moment: Fast and Slow Time in the Information Age. Londres: Pluto Press.

Estalella, Adolfo. 2015. "Prototyping social sciences. Emplacing digital methods", en Helene Snee, Christine Hine, Yvette Morey, Steven Roberts y Hayley Watson (eds.), Digital Methods for Social Sciences. An Interdisciplinary guide to research innovation: 127-141. Houndmills: Palgrave.

Fernández, Sandra. 2015. Sentidos en proceso. Una etnografía sobre prácticas emergentes de producción artístico-tecnológica en contextos urbanos. Tesis doctoral inédita, UNED.

Gill, Rosalind. 2010. "Breaking the silence: The hidden injuries of neo-liberal academia", en Róisín Flood y Rosalind Gill (eds), Secrecy and Silence in the Research Process: Feminist Reflections: 228-244. Londres: Routledge.

Grupo Cultura Urbana UNED. 2016a. «Places of Metropolization. Emerging Social Practices in the Urban Space: The Case of Madrid. Urbanities 6(1).

Grupo Cultura Urbana UNED. 2016b. Cosmópolis. Nuevas maneras de ser urbanos. Barcelona: Gedisa.

Haraway, Donna. 2004. Testigo_Modesto@Segundo_Milenio: HombreHembra@_Conoce_Oncoratón. Barcelona: UOC.

Latour, Bruno. 2001. La esperanza de Pandora. Barcelona: Gedisa.

Law, John. 2004. After Method. Abingdon/N.Y.: Routledge.

Strathern, Marilyn (ed.). 2000. Audit Cultures: Anthropological studies in accountability, ethics and the academy. London: Rutledge.

Tsing, Anna L. 2013. "La selva de las colaboraciones", en Montserrat Cañedo (ed.), Cosmopoliticas. Perspectivas antropológicas de la política: 266-295. Madrid: Trotta. 